\author{
Olga Shtyka, Łukasz Przybysz, Jerzy Sęk \\ Institution Faculty of Process and Environmental Engineering, Technical University of Lodz \\ Wolczanska 177, 90-924 Lodz, Poland, olga.shtyka@edu.p.lodz.pl
}

\title{
TRANSPORT OF EMULSIONS IN GRANULAR POROUS MEDIA DRIVEN BY CAPILLARY FORCE
}

\begin{abstract}
The transport of liquids driven by capillary suction-pressure and balanced by both viscous drag force and gravity acceleration is known as spontaneous imbibition. The prediction of spontaneous imbibition in porous media is of importance due to its relevance as a fundamental phenomenon in numerous industrial technologies as well as in nature. A vast majority of the experimental results and mathematical models concerning the imbibition process of single-phase liquids are considered and analyzed in the literature. The present research focuses on two-phase liquids transport in porous medium driven by capillary force. The penetrating liquids were surfactantstabilized emulsions with the different dispersed phase concentrations. The discussed issues are the influence of porous bed composition and inner phase concentration on the height of an emulsion penetration, which allows to predict the velocity of imbibition process. From a practical point of view, the experimental results give the possibility to evaluate: productivity of granular sorbents applied to recover the environment, efficiency of building materials wetting with multiphase liquids, process of oil-derived pollutants migration in porous media, e.g. soil and other rock structures, etc.
\end{abstract}

\section{Key words}

emulsion, imbibition, kinetics, granular media, pore radius

\section{Introduction}

The liquids transport driven by capillary force and counterbalanced by viscous drag force and gravity acceleration is referred in literature as spontaneous imbibition or wicking [1-4]. The imbibition as a physical phenomenon occurs in porous structures on the condition that adhesion predominates a mutual force of attraction between molecules in a permeating liquid [3-5]. The prediction of the spontaneous imbibition in porous media remains of importance due to its relevance as a fundamental phenomenon in a variety of industrial technologies and in nature: oil recovery and removal of different oil-derivative products from the environment, paper coating, ink penetration process, drug delivery systems, hydrological regime of soil layers, and measuring of contact angle in surface chemistry $[1,2,6]$.

The process of porous media imbibition with such single-phase liquids as water $[1,4,7]$, and different inorganic substances, i.e. dimethyl silicone oil, dodecane, hexadecane, diethyl ether, was experimentally investigated and described in the literature $[3,5,8]$. There are numerous approaches used to describe the single-phase liquids wicking in various porous structures. A great deal of the discussed mathematical models considers the effect of a dynamic contact angle on capillary rise $[4,8,9]$. Another group predicts the spontaneous imbibition in the porous media regarding structural parameters, i.e. porosity, tortuosity and shape of pores [2, 10-12]. A lot of approaches confiders both factors: structure of voids and medium saturation [11, 13, 14]. This allows for the characterization of the imbibition process in a wide range of granular media such as sorbents, soil, silica glasses, and other rocks. The mentioned concepts are appropriate to predict imbibition process in case of various singlephase liquids, while there is lack of experimental results and mathematical models, which predict wicking of multiphase liquids in granular beds.

This research work focuses on the study of the imbibition process in case of oil-in-water emulsions as two-phase dispersions differed by wettability and viscosity. The experiments reported in the paper were undertaken to investigate the kinetics of imbibition in different granular beds in terms of the changes of penetration height as a function of time, i.e. $h_{i m}=f\left(t_{i m}\right)$. The influence of the dispersed phase concentration and the composition of granular media on the kinetics of imbibition, velocity of this process, its instantaneous velocity as well as the maximal height of an emulsion front rise were also considered and discussed. 


\section{Materials and Methods}

In these experiments, the spontaneous imbibition process was investigated experimentally using the wicking test, as described in details in the publication $[6,15]$. The used experimental set-up is schematically represented in Figure 1. A sample of a granular medium (2) was directly immersed with one end into a reservoir with an emulsion (1) with a contact area of $0.00096 \mathrm{~m}^{2}$. The changes of the emulsion mass $m_{i m}$ in a reservoir (1) were registered versus time $t_{i m}$ using an analytical balance (3) as well as the height of its penetration $h_{i m}$ by means of a ruler (4). The time when the mass of the emulsion in a reservoir (1) became steady was assumed as the final time of imbibition process $t_{\max }$. The achieved height of the liquid front at $t_{\max }$ was denoted as the equilibrium height, denoted as $h_{\max }$

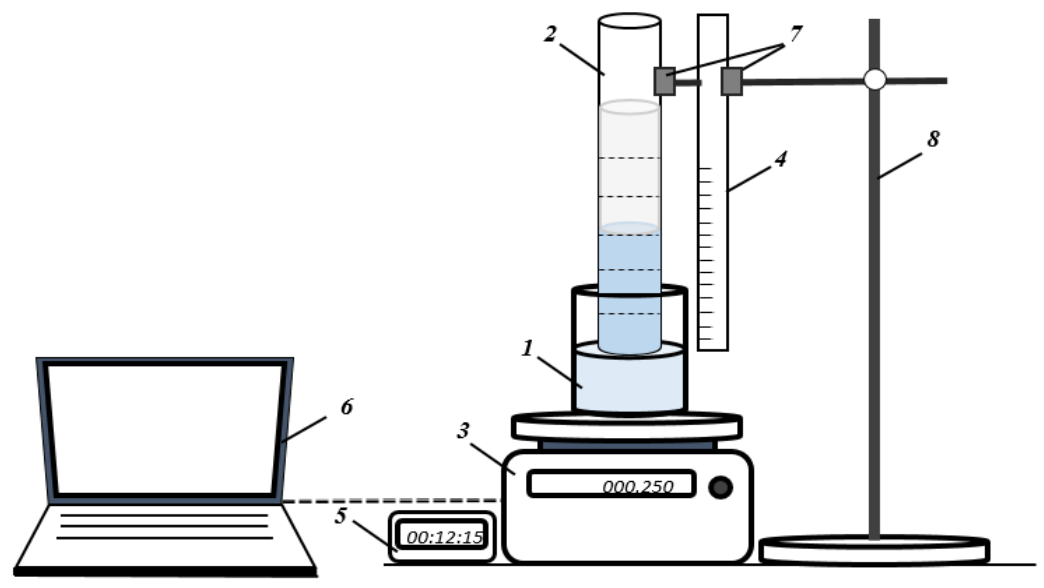

Fig. 1. Schematic illustration of the used experimental set-up for a granular medium: 1 - reservoir with an emulsion; 2 granular bed; 3 - analytical balance; 4 - ruler; 5 - timer CDN TM15; 6 - computer to register data; 7 - fixators; and 8stand. The camera Nikon Coolpix L120 was used to record the changes of the height.

Source: Author's

Three types of oil-in-water emulsions were used in the current experiments. The emulsions were prepared according to the standard procedure. The dispersions differed by the inner phase concentration, which was equal to $10 \mathrm{vol} . \%, 30 \mathrm{vol} . \%$, and $50 \mathrm{vol} . \%$. They were stabilized with 2 vol.\% of a non-ionic surfactant composed of ethoxylated oleic acid (commercial name Rokacet 07), obtained from PCC Exol SA (Poland). The defined volume of the distilled water, as a continuous phase, was mixed with the emulsifier and dispersed phase in a $500 \mathrm{ml}$ beaker with a diameter $d_{b k}$ of $0.08 \mathrm{~m}$. The immiscible phases and surfactant were mixed by means of a high shear laboratory homogenizer with revolution of $12000 \mathrm{~min}^{-1}$ during $600 \mathrm{~s}$.

Microscopic images analysis of the prepared emulsions was carried out by means of Microscope Leica DMI3000B with a Lumenera Infinity1 camera. The distribution of the dispersed droplet size in emulsions is shown in Fig. 2. According to the results, the diameters of dispersed oil droplets in the prepared emulsions were in the range 1$20 \mu \mathrm{m}$, while a majority of them (75-80\%) had a size of $2-10 \mu \mathrm{m}$.

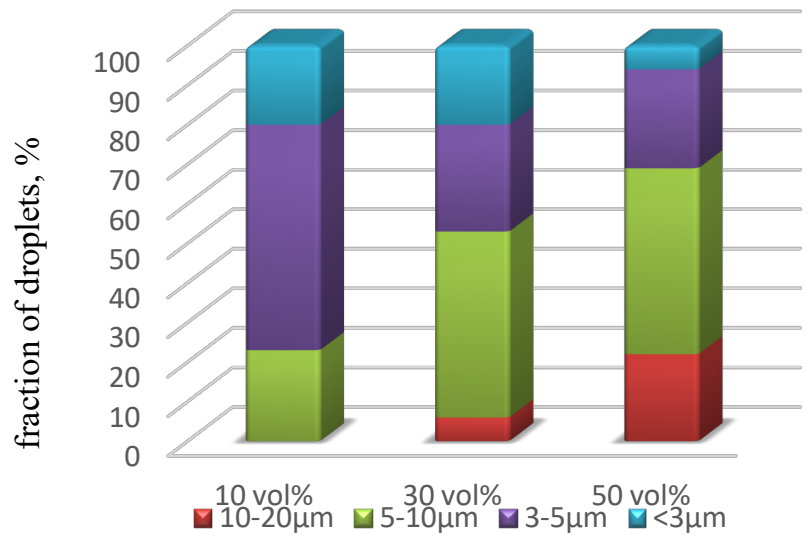

Fig. 2. Distribution of the dispersed droplet size in emulsions stabilized by the surfactant in concentration $\varphi_{s}$ of 2 vol.\%. Source: Author's 
The viscosity was measured using a shear rheometer Bohlin CVO120 (Malvern Instruments, UK). The density was determined using the picnometric method. The surface tension was measured using a tensiometer KRÜSS K12 (KRÜSS GmbH, Germany). The physicochemical properties of the emulsions components are shown in Table 1.

Table 1. Properties of emulsions components $\left(T=23 \pm 1^{\circ} \mathrm{C}\right)$

\begin{tabular}{|l|c|c|c|c|}
\hline Type of liquid & $\begin{array}{c}\text { Density } \\
\left(\mathbf{k g} / \mathbf{m}^{\mathbf{3}}\right)\end{array}$ & $\begin{array}{c}\text { Viscosity } \\
(\mathbf{m P a} \cdot \mathbf{s})\end{array}$ & $\begin{array}{c}\text { Surface tension } \\
(\mathrm{mN} / \mathrm{m})\end{array}$ & $\begin{array}{c}\text { HLB } \\
(-)\end{array}$ \\
\hline Dispersed phase & $922.1 \pm 0.6$ & $53.12 \pm 1.44$ & $32.2 \pm 1.7$ & - \\
\hline Rokacet O7 & $908.0 \pm 2.7$ & $50.21 \pm 0.62$ & $36.2 \pm 1.8$ & 10.6 \\
\hline
\end{tabular}

Source: Author's

The porous medium was represented by a granular bed consisting of spherical granules, and characterized by the oleophilic/hydrophilic property. The beads were produced and obtained from "Alumetal-Technik" (Poland). The used porous media differed by a size of the particles, and their diameters ranged from 100-800 $\mu \mathrm{m}$. The parameters of the granular media used are provided in Table 2.

Table 2. Parameters of the granular media

\begin{tabular}{|l|c|c|c|}
\hline $\begin{array}{c}\text { Type of } \\
\text { medium }\end{array}$ & $\begin{array}{c}\text { Range of beads } \\
\text { diameter }(\boldsymbol{\mu m})\end{array}$ & $\begin{array}{c}\text { Average diameter } \\
\text { of beads, } \boldsymbol{d}_{\boldsymbol{a}}(\boldsymbol{\mu \mathrm { m } )}\end{array}$ & Porosity, $\boldsymbol{\varepsilon}$ \\
\hline GS 100 & $100-200$ & $180 \pm 10.9$ & $0.35 \pm 0.010$ \\
\hline GS 200 & $200-300$ & $245 \pm 12.3$ & $0.36 \pm 0.011$ \\
\hline GS 600 & $600-800$ & $650 \pm 9.2$ & $0.37 \pm 0.013$ \\
\hline
\end{tabular}

Source: Author's

All experiments were conducted at $23 \pm 1^{\circ} \mathrm{C}$ and atmospheric pressure. Three independent replications were carried out for each experiment, and results were presented as their mean values.

\section{Result and Discussion}

The results concerning the change of the emulsion penetration height $h_{i m}$ as a function of time $t_{i m}$ for the different granular beds are represented in Figure 3 . The used granular beds were composed of spherical grains with sizes in the ranges of 100-200 $\mu \mathrm{m}, 200-300 \mu \mathrm{m}$, and 600-800 $\mu \mathrm{m}$. The data were obtained for emulsions with the different dispersed phase concentrations of 10 vol\%, 30 vol\%, and 50 vol\%.

As shown in Figure 3, the emulsions differ significantly by the height of penetration, thus a fraction of beads size and consequently, radii of pores in a granular bed causes the strong influence on the height of the emulsion front rise. The hydraulic radius of pores $r_{h}$ was calculated according to Kozeny-Carman theory as a relation between medium porosity and average diameter of beads [16]. Thus, it was $16.2 \pm 0.16 \mu \mathrm{m}$ for a medium with particles diameter $d_{b}$ in a range of 100-200 $\mu \mathrm{m}, 23.0 \pm 0.38$ and $63.6 \pm 0.18 \mu \mathrm{m}$ for GS 200 and GS 600 , respectively. To compare used granular beds, all emulsions tended to wick higher in case of medium with lower value of $r_{h}$. In contrast, the dispersed phase concentration is recognized initially as a less important factor. 

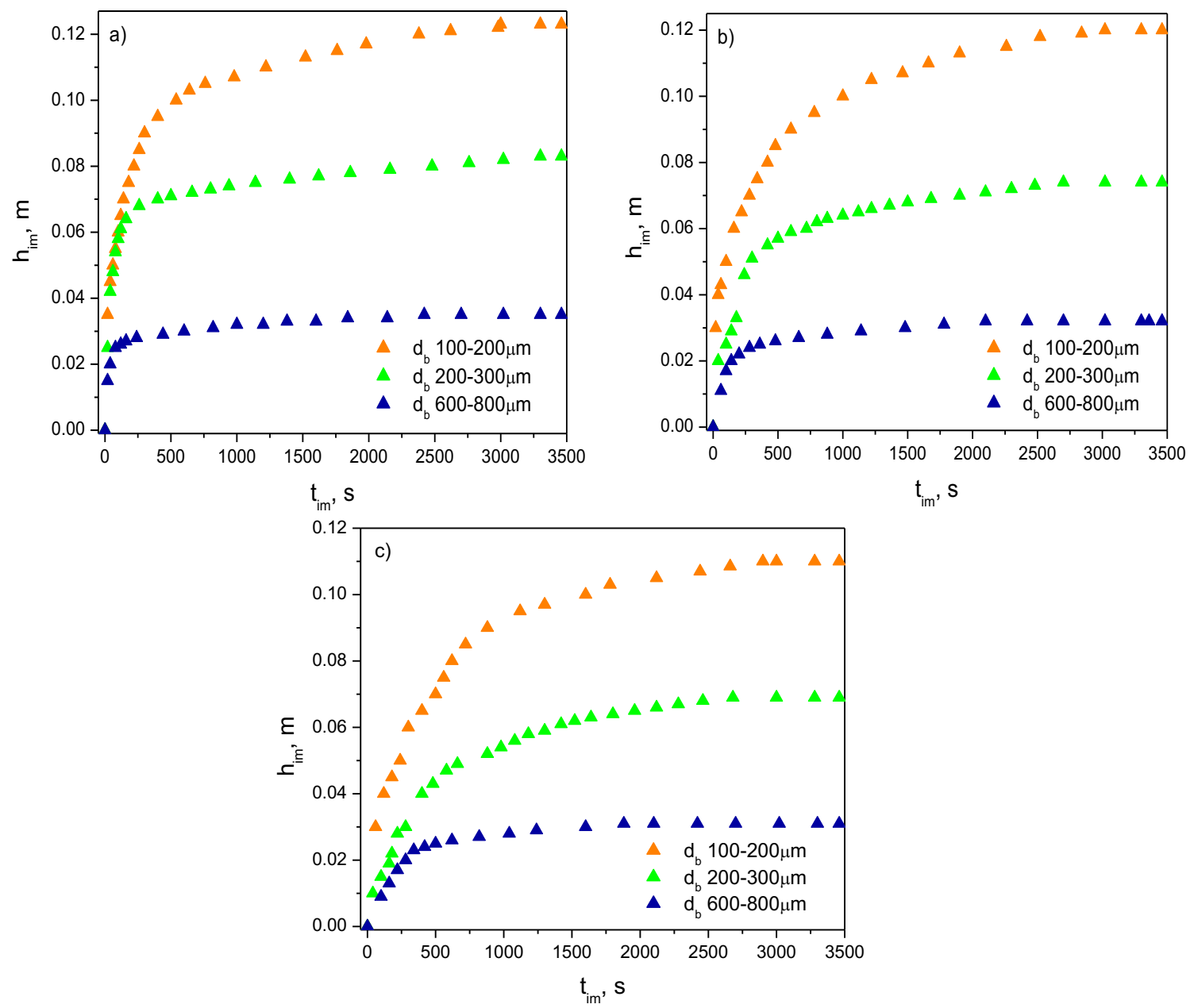

Fig. 3. Changes of emulsion front height $h_{i m}$ versus time $t_{i m}$ during the imbibition process in the different granular media in case of: $a-10 \%$ emulsions; $b-30 \%$ emulsions; $c-50 \%$ emulsions.

Source: Author's

The experimental data allowed to define the maximal height of emulsions penetration in the different granular media, and the results are shown in Table 3.

Table 3. The maximal height $h_{\max }$ of the imbibed emulsions

\begin{tabular}{|c|c|c|c|}
\hline \multirow{2}{*}{$\begin{array}{c}\text { Type of } \\
\text { medium }\end{array}$} & \multicolumn{3}{|c|}{ Dispersed phase fraction, $\boldsymbol{\varphi}_{\boldsymbol{d}}$} \\
\cline { 2 - 4 } & $\mathbf{1 0}$ vol\% & $\mathbf{3 0}$ vol\% & $\mathbf{5 0 ~ v o l \%}$ \\
\hline GS 100 & 0.123 & 0.120 & 0.110 \\
\hline GS 200 & 0.083 & 0.074 & 0.069 \\
\hline GS 600 & 0.035 & 0.032 & 0.031 \\
\hline
\end{tabular}

Source: Author's

The maximal height tends to decrease slightly, i.e. maximum up to $17 \%$, with the enlarging of the dispersed phase concentration. Such tendency was observed for porous beds with different compositions. The experimental results shown that the highest values of $h_{\max }$ were obtained in case of the granular medium composed of beads with $d_{b}$ in a range of $100-200 \mu \mathrm{m}$, i.e. $0.123,0.12$, and $0.11 \mathrm{~m}$ for $10 \%, 30 \%$, and $50 \%$ emulsions, respectively. Consequently, the lowest values were derived for the spontaneous imbibition process in a granular medium with $d_{b}$ of $600-800 \mu \mathrm{m}$.

The changes of the imbibition velocity as $v_{i m h}=f\left(t_{i m}\right)$ on the base of height values in the used granular beds are shown in Figure 4. 

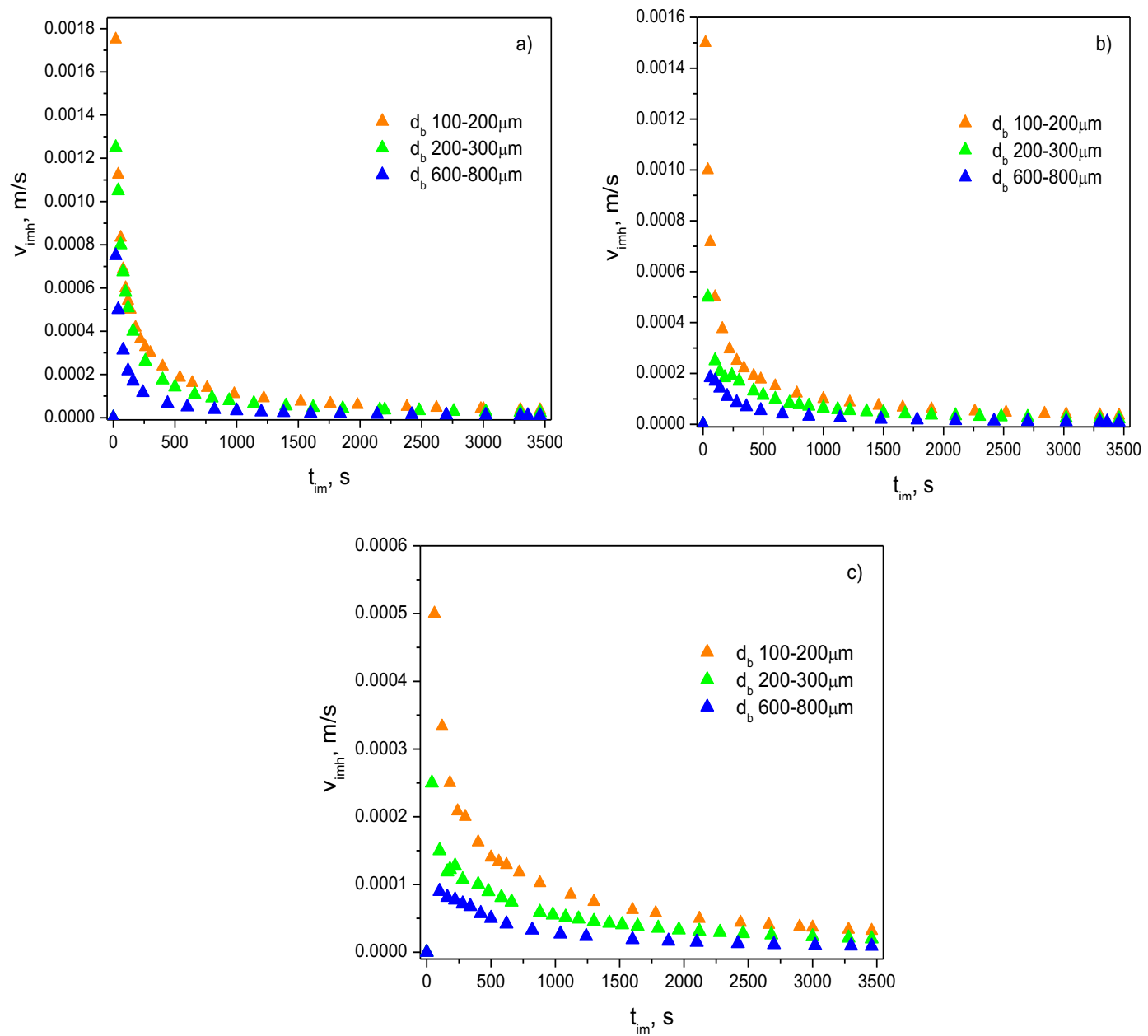

Fig. 4. Changes of the imbibition velocity $v_{i m h}$ versus time $t_{i m}$ for: $a-10 \%$ emulsions; $b-30 \%$ emulsions; $c-50 \%$ emulsions Source: Author's

The imbibition velocity $v_{i m h}$ tends to rise rapid and after that decreases as shown on the graphs. It can be explained by growth of the counterbalancing force, i.e. gravity acceleration, due to increase of an imbibied emulsion mass. The values of the maximal velocity $v_{\max }$ of the wicking process and time $t\left(v_{\max }\right)$, when it was reached, are represented in Table 4.

Table 4. The maximal velocity $v_{\max }$ of the imbibition process

\begin{tabular}{|l|c|c|c|c|c|c|}
\hline \multirow{2}{*}{$\begin{array}{l}\text { Dispersed phase } \\
\text { concentration, } \boldsymbol{\varphi}_{\boldsymbol{d}}\end{array}$} & \multicolumn{2}{|c|}{ GS 100 } & \multicolumn{2}{c|}{ GS 200 } & \multicolumn{2}{c|}{ GS 600 } \\
\cline { 2 - 7 } & $\boldsymbol{v}_{\text {max }}, \mathbf{m} / \mathbf{s}$ & $\boldsymbol{t}\left(\boldsymbol{v}_{\text {max }}\right), \mathbf{s}$ & $\boldsymbol{v}_{\text {max }}, \mathbf{m} / \mathbf{s}$ & $\boldsymbol{t}\left(\boldsymbol{v}_{\max }\right), \mathbf{s}$ & $\boldsymbol{v}_{\text {max }}, \mathbf{m} / \mathbf{s}$ & $\boldsymbol{t}\left(\boldsymbol{v}_{\max }\right), \mathbf{s}$ \\
\hline 10 vol\% & 0.00175 & 20 & 0.00125 & 20 & 0.00075 & 20 \\
\hline 30 vol\% & 0.00150 & 20 & 0.00050 & 40 & 0.00018 & 60 \\
\hline 50 vol\% & 0.00050 & 40 & 0.00025 & 40 & 0.00009 & 100 \\
\hline
\end{tabular}

Source: Author's

In all investigated cases, the highest maximal velocity values were obtained in case of the dispersions with the lowest inner phase concentration. It can be explained by the difference in viscosity, and lower value is less influence of the viscous drag force. Thus, the prepared emulsions have the following viscosities: for $10 \%$ it was equal to $6.1 \mathrm{mPa} \cdot \mathrm{s}$, in case of $30 \%$ this parameter was near $14.8 \mathrm{mPa} \cdot \mathrm{s}$, and the largest value was obtained for $50 \%$ dispersion, i.e. $48.4 \mathrm{mPa} \cdot \mathrm{s}$.

As shown in Table 4, it is equal to $0.00175 \mathrm{~m} / \mathrm{s}$ for the porous bed with $d_{b}$ of $100-200 \mu \mathrm{m}, 0.00125 \mathrm{~m} / \mathrm{s}$ for 200 $300 \mu \mathrm{m}$, and consequently, $0.00075 \mathrm{~m} / \mathrm{s}$ for $600-800 \mu \mathrm{m}$. To compare, the lowest values of $v_{\text {imh }}$ were observed for $50 \%$ emulsions in all used granular beds. The maximal velocity was registered at $t_{i m}=20 \mathrm{~s}$ for $10 \%$ emulsions, 
however it tends to growth with increase of the inner phase concentration. The enlargement of grains size and consequently, hydraulic radius of pores causes the increase of time needed to obtain the maximal velocity, i.e. up to $20 \mathrm{~s} \leq t_{i m} \leq 100 \mathrm{~s}$ (Table 4).

The changes of instantaneous velocity $v_{i n h}$ with time $t_{i m}$ was additionally calculated relating to average velocity of the imbibition process. It represented as velocity of a liquid in motion at the specific point of time process. The results of the instantaneous velocity variations are performed in Figure 5.

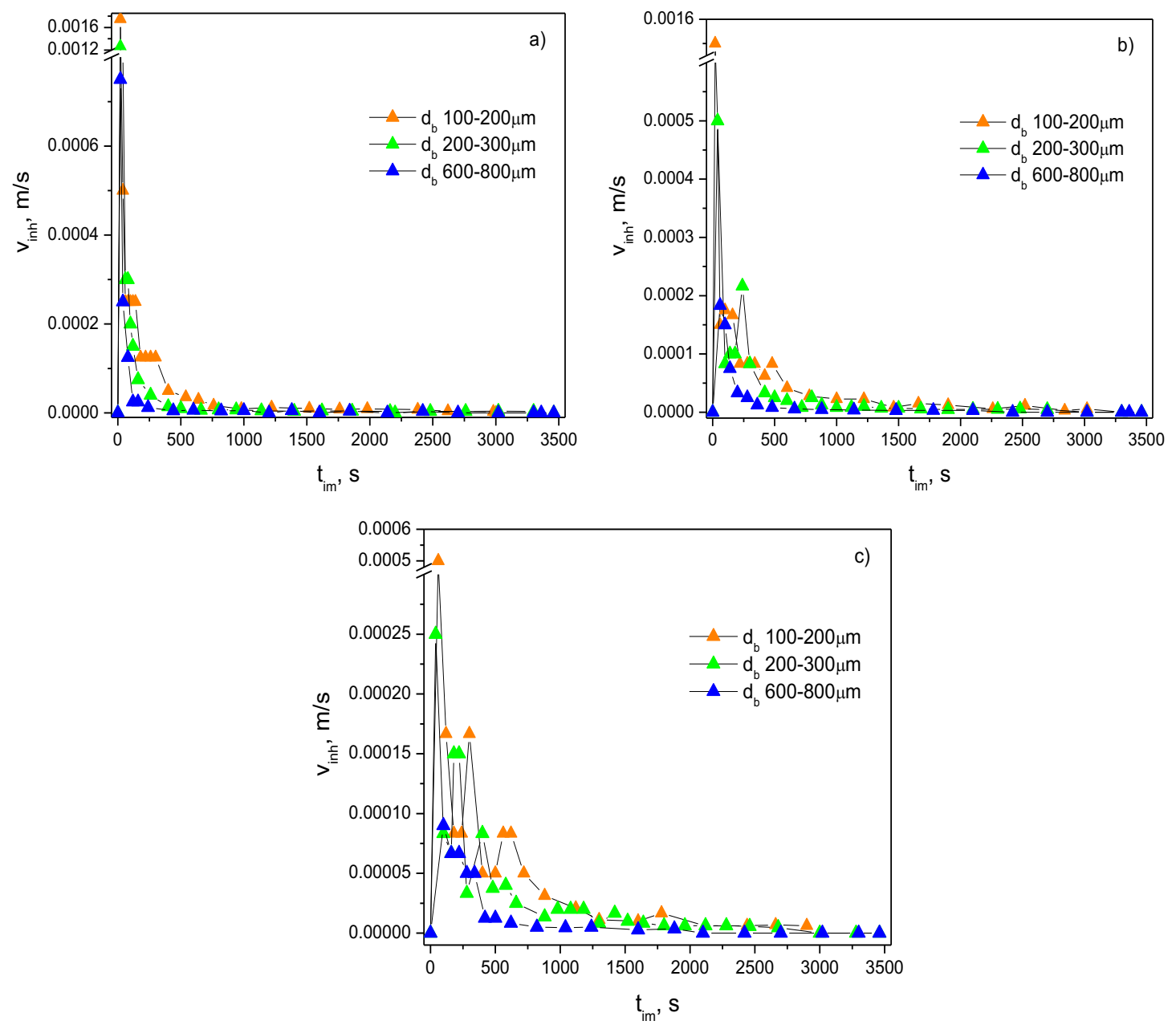

Fig. 5. Changes of the instantaneous imbibition velocity $v_{\text {inh }}$ versus time $t_{i m}$ for: $a-10 \%$ emulsions; $b-30 \%$ emulsions; $c-$ $50 \%$ emulsions.

Source: Author's

As show in Figure 5a, the higher instantaneous imbibition velocity was obtained for $10 \%$ emulsions in all granular beds investigated as in the case of the imbibition velocity $v_{i m h}$. In contrast, the lowest values were derived for $50 \%$ dispersions. According to the results, the stronger influence of a bed composition is also observed for emulsions with the inner phase concentration of $50 \mathrm{vol} \%$. In case of $10 \%$ emulsions, the instantaneous imbibition velocity becomes almost the same after $t_{i m}=800 \mathrm{~s}$ (Fig. 5a).

To conclude, the emulsions imbibition in terms of the penetration height depends considerably on the composition of a granular bed. On the one hand, the dispersed phase concentration causes less significant influence on the height of an imbibed emulsion wicking in a granular media. On the other hand, the composition of the dispersed phase effects the imbibition velocity as well as instantaneous one.

\section{Summary and conclusions}

The discussed issues were the influence of porous bed composition and dispersed phase concentration on the height of emulsion penetration, which allows to predict velocity of the imbibition process and the maximal height 
of dispersion rise in a granular bed. According to results, the height of an emulsion front permeation in a granular medium forced by the capillary pressure depends more strongly on the composition of this bed than on the initial concentration of the dispersed phase. However, the effect of a porous medium structure was more considerable in case of $50 \%$ dispersions. From a practical point of view, the experimental results give the possibility to predict the productivity of granular sorbents applied to recovery the environment, the building materials wetting with dispersions, i.e. paints, antifungal liquid, and pollutants migration in various porous media, i.e. soil layers, sands and other rock structures.

\section{References}

[1] R. Masoodi, K.M. Pillai, Darcy's law-based model for wicking in paper-like swelling porous media, AlChE Journal 56 (2010) 2257-2267.

[2] J. Cai, X. Hu, D. S. Standnes, L. You, An analytical model for spontaneous imbibition in fractal porous media including gravity, Colloids Surf., A 414 (2012) 228-233.

[3] R. Masoodi, K.M. Pillai, P.P. Varanasi, Darcy's law based models for liquid absorption in polymer wicks, AlChE Journal 53 (2007) 2769-2782.

[4] A. Hamraoui, T. Nylander, Analytical approach for the Lucas-Washburn equation, J. Colloid Interface Sci. 250 (2002) 415-421.

[5] B.V. Zhmud, F. Tiberg, K. Hallstensson, Dynamics of capillary rise, J. Colloid Interface Sci. 228 (2000) 263-269.

[6] O. S. Shtyka, M. M. Błaszczyk, J. P. Sęk, Analysis of emulsions concentration changes during imbibition in porous sorbents, IJEST 13 (2016) 2401-2414.

[7] H.T. Xue, Z.N. Fang, Y. Yang, J.P. Huang, L.W. Zhou, Contact angle determined by spontaneous dynamic capillary rises with hydrostatic effects: Experiment and theory, Chem. Phys. Lett. 432 (2006) 326-330.

[8] R.M. Digilov, Capillary rise of a non-Newtonian power law liquid: impact of the fluid rheology and dynamic contact angle, Langmuir 24 (2008)13663-13667.

[9] M. Hilpert, Liquid withdrawal from capillary tubes: explicit and implicit analytical solution for constant and dynamic contact angle, J. Colloid Interface Sci. 351 (2010) 267-276.

[10] H.Y. Zhao, K.W. Li, A fractal model of production by spontaneous water imbibition, Latin American and Caribbean Petroleum Engineering Conference (2009).

[11] J. Cai, E. Perfect, C.L. Cheng, X. Hu, Generalized Modeling of Spontaneous Imbibition Based on Hagen-Poiseuille Flow in Tortuous Capillaries with Variably Shaped Apertures, Langmuir 30 (2014) 5142-5151.

[12] C.L. Cheng, E. Perfect, B. Donnelly et al., Rapid imbibition of water in fractures within unsaturated sedimentary rock, Adv. Water Resour. 77 (2015) 82-89.

[13] K.W. Li, R.N. Horne, An analytical scaling method for spontaneous imbibition in gas-water-rock systems, SPE Journal 9 (2004) 322-329.

[14] K.W. Li, R.N. Horne, Generalized Scaling Approach for Spontaneous Imbibition: An Analytical Model, SPE Journal 9 (2006) doi.org/10.2118/77544-PA.

[15] O. Shtyka, J. Sęk, M. Błaszczyk, S. Kacprzak, Investigation into hydro- and oleophilic porous medium saturation with two-phase liquids during the imbibition process, Inż. Ap. Chem. 55 (2016) 36-374.

[16] J.S. Kowalski, Inżyneria materiałów porowatych, Wydawnictwo Politechniki Poznańskiej, Poznan (2004). 